\title{
Growth mode and matrix effects on the magneto-optical activity of ultrathin films
}

\author{
J. L. Menéndez, G. Armelles, A. Cebollada, ${ }^{\text {a) }}$ and C. Quintana \\ Instituto de Microelectrónica de Madrid, (CNM-CSIC), Isaac Newton, 8-PTM 28760 Tres Cantos, Madrid, \\ Spain \\ D. Ravelosona and C. Chappert \\ Institut d'Electronique Fondamentale, Unité de Recherche Asociée CNRS 022, Université Paris \\ Sud 91405 Orsay Cedex, France \\ F. Peiró and A. Cornet \\ Electronic Materials and Engineering, Facultat de Física, Universitat de Barcelona, Av. Diagonal 645-647, \\ E-08028 Barcelona, Spain
}

(Received 1 March 2002; accepted for publication 1 July 2002)

\begin{abstract}
The magneto-optical activity of Fe nanoparticulate systems is studied in this work as a function of the matrix that surrounds the particles and the Fe concentration in the effective layer. It is shown that the magneto-optical activity is not linear or proportional to the amount of Fe even at low coverages when the matrix surrounding the particles is an insulator. As a conclusion, it is also demonstrated that the magneto-optical response of a magnetic ultrathin film system strongly depends on its growth mode, with modifications of up to $100 \%$ in its magneto-optical activity. (C) 2002 American Institute of Physics. [DOI: 10.1063/1.1502913]
\end{abstract}

The study of the magneto-optical (MO) activity of nanostructured systems has great technological interest as their possible application for magnetic recording has been suggested. ${ }^{1}$ From a basic point of view, various MO effects have been used to investigate properties such as the electronic structure ${ }^{2}$ and magnetic moments ${ }^{3}$ in magnetic systems. When the amount of deposited material is in the range of a few atomic layers, where the incident light probes the whole structure, it is generally considered that the MO activity is linear with the amount of magnetic material deposited, and deviations from that linear behavior are usually attributed to different reasons such as novel MO effects ${ }^{4}$ or changes in the electronic structure. ${ }^{5}$ On the other hand, large decreases in the magnetic moments of magnetic systems are often observed in MO measurements at low coverages. ${ }^{6}$ Despite this effort, no study has considered the influence that the growth mode and the matrix in the case of nanoparticulate materials exert on the MO activity of a system. The aim of this work is to study this influence.

Due to the ex situ character of all the MO techniques available, the experimental part of this work will be restricted to magnetic structures embedded in both metallic and insulating matrices. Thicknesses were determined by combination of atomic force microscopy (AFM) and transmission electron microscopy (TEM) measurements. As a first step, the MO activity of $\mathrm{Fe}$ islands embedded in different matrices was studied in order to consider the influence of the optical character of the matrix on the MO activity of the system. Fe islands were embedded in two different matrices: a metallic one, $\mathrm{Pt}$, and an insulator, $\mathrm{MgO}$. In the former case, the $\mathrm{Fe}(110)$ islands were sputtered at $700^{\circ} \mathrm{C}$ on an $\mathrm{Al}_{2} \mathrm{O}_{3}(0001)$ substrate. This deposition temperature gives

\footnotetext{
a) Author to whom correspondence should be addressed; electronic mail: alfonso@imm.cnm.csic.es
}

rise to the formation of islands. ${ }^{7}$ The islands were then cooled down to room temperature and a $25 \AA$ thick Pt capping layer was deposited to cover them. In the second case, a $\mathrm{GaAs}(001)$ substrate was used and a $100 \AA$ layer of $\mathrm{MgO}(100)$ was deposited at $400^{\circ} \mathrm{C}$ by laser ablation. $\mathrm{Fe}(100)$ islands were then deposited at $700^{\circ} \mathrm{C}$ on the $\mathrm{MgO}$ layer and cooled down to $400^{\circ} \mathrm{C}$ to deposit another $100 \AA$ $\mathrm{MgO}(100)$ layer on top by laser ablation. The dimensions of the islands were always much smaller than the wavelength of the light used in the MO measurements.

The polar (transverse) Kerr effect was measured applying in the polar (transverse) configuration a maximum field of $1.5 \mathrm{~T}(0.2 \mathrm{~T})$. As the $\mathrm{Fe}$ islands were small compared to the wavelength of the light, it was possible to apply effective medium theories to describe optically and magneto-optically the nanoparticulate layers. In the systems considered in this work it is reasonable to assume the presence of very small interfacial layers between $\mathrm{Fe}$ and $\mathrm{Pt}$ or $\mathrm{MgO}$ which have not been considered due to the sizes of the islands relative to these layers. Therefore the dielectric tensors of bulk Fe, Pt, and $\mathrm{MgO}$ have been used. The effective medium theory used was a self-consistent one already described in Ref. 8, the transfer matrix formalism ${ }^{9}$ being used to perform the MO calculations.

The Kerr activity was studied at $800 \mathrm{~nm}$, although spectra from 300 to $800 \mathrm{~nm}$ were measured in both polar and transverse configurations. Due to the high absorption of the GaAs substrate at $400 \mathrm{~nm}$, it is critical to have a careful characterization of the thicknesses of the buffer and capping layers in order to predict the MO activity of the structures grown on this substrate around this wavelength, as small deviations in their values may lead to important mixings of the Kerr rotation and ellipticity. ${ }^{10}$ For this reason, it is more convenient to study the MO activity at wavelengths far from $400 \mathrm{~nm}$. 


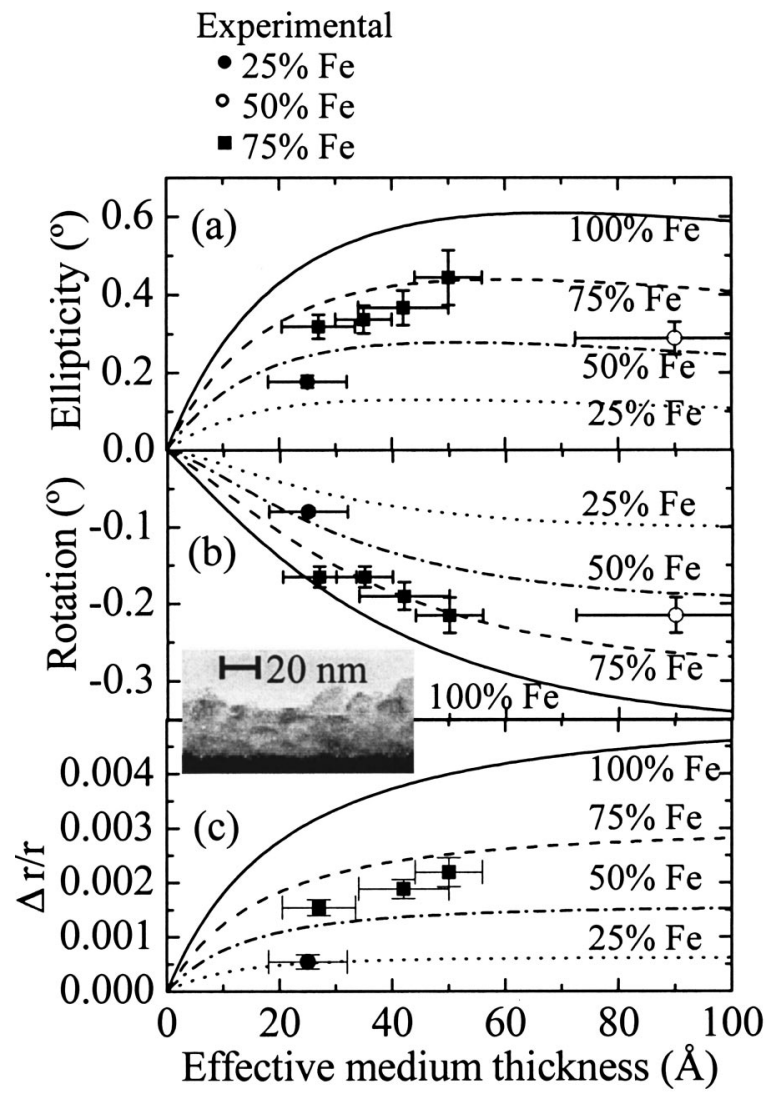

FIG. 1. MO Kerr activity of Fe islands embedded in a Pt matrix in the polar, (a) ellipticity and (b) rotation, and (c) transverse configurations. Lines (symbols) correspond to calculations (experimental data). The inset is a TEM cross section showing the $\mathrm{Fe}$ islands.

In selected samples, TEM characterization was carried out using CM30-SuperTwin and CM200-FEG microscopes, reaching a resolution of $0.2 \mathrm{~nm}$.

In Fig. 1, the calculated MO activity in the polar [Figs. 1(a) $-1(\mathrm{~b})$ ] and transverse [Fig. 1(c)] configurations as a function of the thickness of the effective layer, formed by $\mathrm{Fe}$ particles capped with $\mathrm{Pt}$, is presented for several concentrations of Fe. The MO activity increases with both the thickness and the amount of $\mathrm{Fe}$ in the layer. The experimental data, represented by the symbols, show a good agreement with the theoretical predictions. It can also be shown how the $\mathrm{MO}$ activity in a $3 \AA$ effective layer formed by $\mathrm{Fe}$ and $\mathrm{Pt}$ is proportional and, mainly linear, to the Fe concentration.

So far, this behavior agrees with that usually expected, that is, the higher amount of magnetic material, the higher MO activity. However, the situation is different when the matrix is an insulator instead of a metal. In the case of $\mathrm{Fe}$ islands surrounded by $\mathrm{MgO}$, the calculated ellipticity [Fig. 2(a)] is negative at low concentrations, regardless the effective layer thickness, changing sign at higher concentrations and increasing its value up to $100 \% \mathrm{Fe}$ concentration. This clearly shows how the MO response of Fe particles surrounded by an insulator is clearly different from the expected linear behavior. However, in this particular case, both the polar Kerr rotation [Figs. 2(a)-2(b)] and the transverse Kerr effect [Fig. 2(c)] increase when the Fe concentration is increased. Again, the experimental data agree with the theoretical predictions. However, it was not possible to perform transverse Kerr measurements in these samples as the availDownloaded 14 Jun 2010 to 161.111.235.252. Redistribution subje

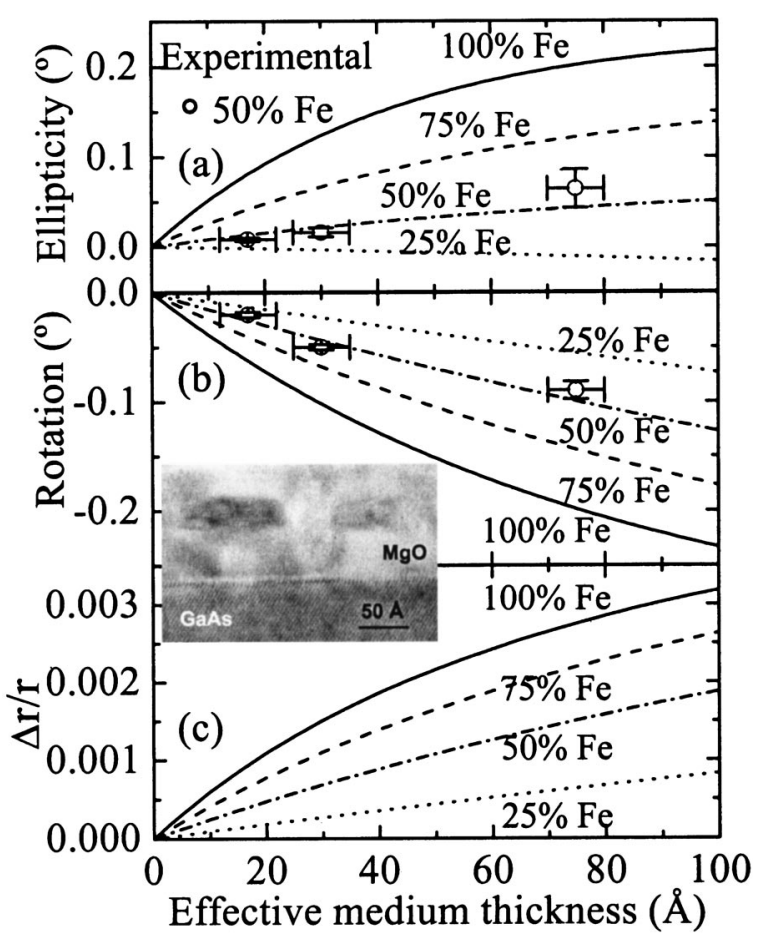

FIG. 2. MO Kerr activity of Fe islands embedded in a $\mathrm{MgO}$ matrix in the polar, (a) ellipticity and (b) rotation, and (c) transverse configurations. Lines (symbols) correspond to calculations (experimental data). The inset is a TEM cross section, showing the Fe islands.

able magnetic field was not enough to reach the magnetic saturation.

If a $3 \AA$ effective layer made of $\mathrm{Fe}$ and $\mathrm{MgO}$ is considered and the concentration of $\mathrm{Fe}$ in the layer varied, the polar [Figs. 3(a)-3(b)] and transverse [Fig. 3(c)] Kerr effects are

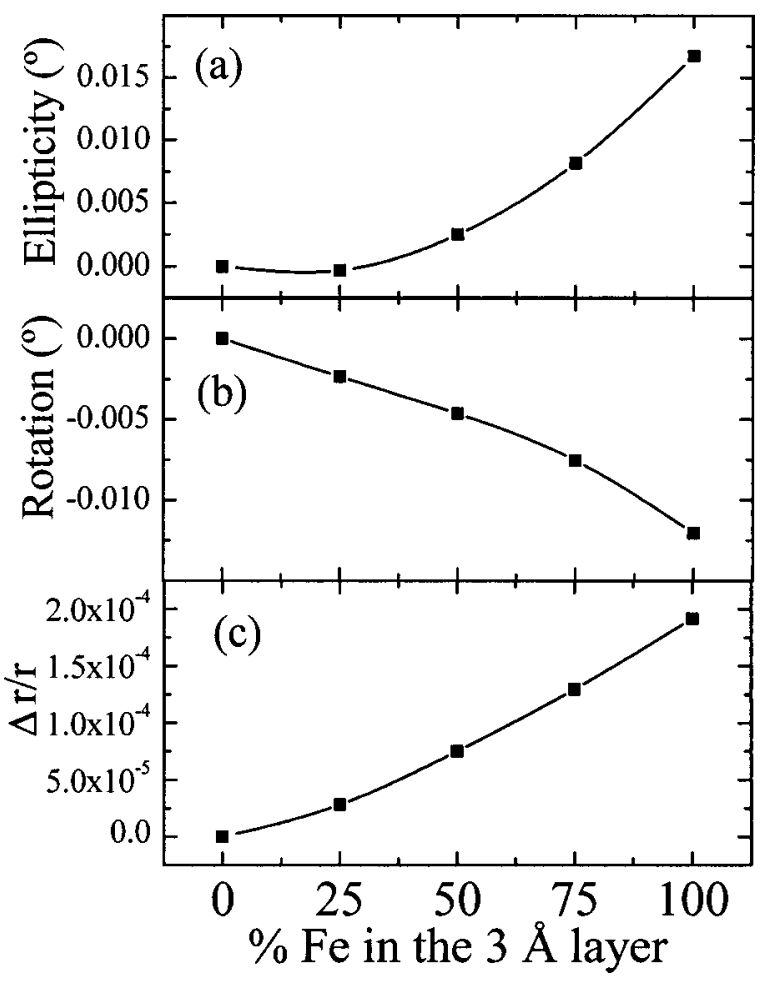

FIG. 3. Evolution of the MO Kerr activity as a function of the Fe content in a, $\mathrm{Fe}+\mathrm{MgO}, 3 \AA$ thick effective medium in the polar, (a) ellipticity and (b) rotation, and (c) transverse configurations.

to AIP license or copyright; see http://apl.aip.org/apl/copyright.jsp 


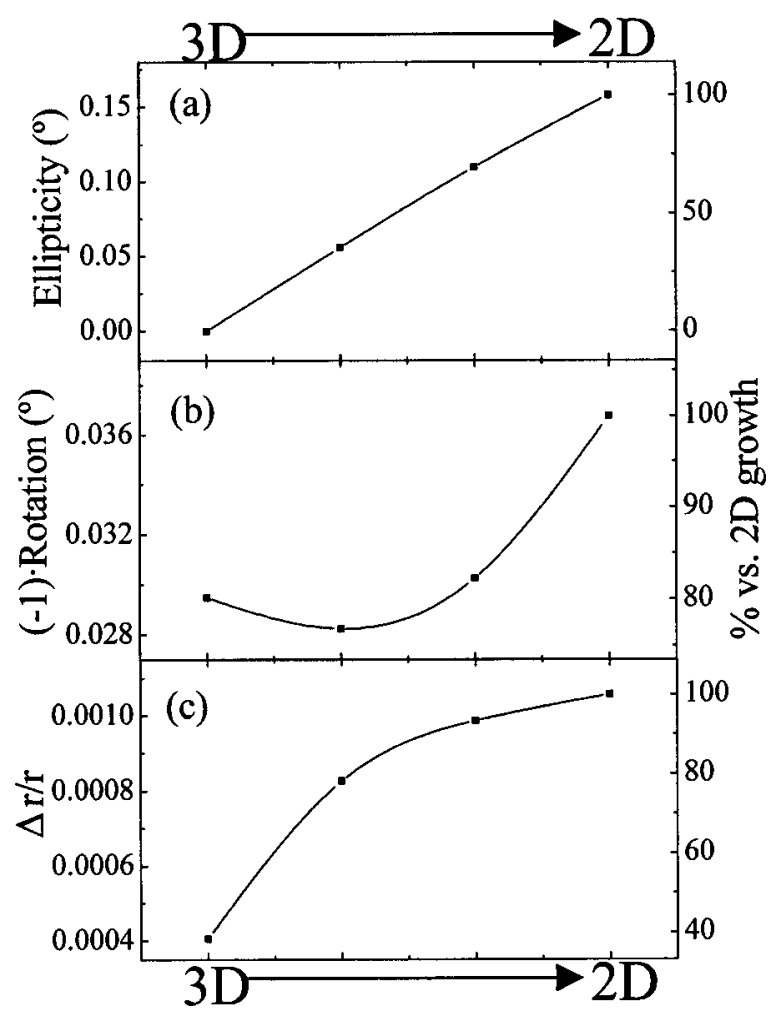

FIG. 4. Calculated Kerr activity in the polar, (a) ellipticity and (b) rotation, and (c) transverse configuration as a function of the growth mode.

no longer linear with the Fe concentration in the layer, although they are proportional to it and the polar Kerr ellipticity is not even proportional, showing a negative value at low concentrations, then a minimum, changing sign and then increasing its value up to the pure Fe layer.

Provided that $\mathrm{MgO}$ and vacuum are optically similar, this nonlinear behavior is expected to be also present in $\mathrm{Fe}$ structures surrounded by vacuum as in in situ characterization. All the theoretical calculations shown in Fig. 4 were made for $5 \AA$ of $\mathrm{Fe}$ deposited, the only difference being the way that $\mathrm{Fe}$ is distributed on the substrate. In a $2 \mathrm{D}$ growth, the magnetic medium will be a continuous $5 \AA \mathrm{Fe}$ layer, in the case of a 3D growth, the magnetic system will be formed by $\mathrm{Fe}$ islands higher than $5 \AA$ and surrounded by vacuum: an effective medium of $\mathrm{Fe}$ and vacuum. If the MO activity in this ultrathin film did not depend on the system growth mode, then the calculated MO activity should be the same in all the configurations studied. However, the calculations show that the polar Kerr ellipticity [Fig. 4(a)] is almost zero in a strong 3D growth, the polar Kerr rotation [Fig. 4(b)] decreases up to a $60 \%$ with respect to the rotation in a $2 \mathrm{D}$ growth, and in the transverse configuration [Fig. 4(c)] the activity decreases up to a $40 \%$ of that in the $2 \mathrm{D}$ case.

In summary, it has been shown how the growth mode of magnetic systems dramatically affects their expected MO activity, which has important consequences on the use of MO techniques as magnetometry tools, especially in low dimensional magnetic systems.

Financial support from the Spanish Commission of Science and Technology is gratefully acknowledged.

${ }^{1}$ M. Mansuripur, in The Physical Principles of Magneto-Optical Recording (Cambridge University Press, Cambridge, 1995).

${ }^{2}$ J. Schoenes, J. Alloys Compd. 275-277, 148 (1998).

${ }^{3}$ Y. B. Xu, E. T. M. Kernohan, D. J. Freeland, A. Ercole, M. Tselepi, and J. A. C. Bland, Phys. Rev. B 58, 890 (1998).

${ }^{4}$ S.-Y. Wang, Z.-C- Shen, W.-M. Zheng, R.-J. Zhang, Y.-X. Zheng, S.-MZhou, and L.-Y. Chen, Physica B 279, 109 (2000).

${ }^{5}$ H. Sato and T. Miyazaki, Jpn. J. Appl. Phys., Part 1 38, 6694 (1999).

${ }^{6}$ T. Nawrath, H. Fritzche, F. Klose, J. Nowikow, and H. Maletta, Phys. Rev. B 60, 9525 (1999).

${ }^{7}$ J. L. Menéndez, G. Armelles, and C. Quintana y A. Cebollada, Surf. Sci. 482-5, 1135 (2001).

${ }^{8}$ J. L. Menéndez, B. Bescós, G. Armelles, R. Serna, J. Gonzalo, R. Doole, A. K. Petford-Long, and M. I. Alonso, Phys. Rev. B 65, 205413 (2002).

${ }^{9}$ M. Schubert, Phys. Rev. B 53, 4265 (1996).

${ }^{10}$ S. Visnovsky, M. Nyvlt, V. Prosser, R. Lopusnik, R. Urban, J. Ferré, G. Pénnisard, D. Renard, and R. Krishnan, Phys. Rev. B 52, 1090 (1995). 